\title{
Skin endometriosis: A case report and review of the literature
}

\author{
ANDREEA-MARIANA MATEI ${ }^{1,2 *}$, ANA-MARIA DRAGHICI-IONESCU ${ }^{1 *}$, MIRELA CIOPLEA $^{3,4}$, \\ SABINA ANDRADA ZURAC ${ }^{3,4}$, DANIEL BODA ${ }^{5,6}$, IOANA SERBAN ${ }^{6}$, CONSTANTIN CARUNTU $^{2,5}$, \\ MIHAELA ADRIANA ILIE ${ }^{6}$ and LÁSZLÓ FEKETE GYULA ${ }^{7}$ \\ ${ }^{1}$ Department of Dermatology, Colentina Clinical Hospital, 020125 Bucharest; \\ Departments of ${ }^{2}$ Physiology, ${ }^{3}$ Pathology, 'Carol Davila' University of Medicine and Pharmacy, 050474 Bucharest; \\ ${ }^{4}$ Department of Pathology, Colentina Clinical Hospital, 020125 Bucharest; ${ }^{5}$ Department of Dermatology, \\ 'Prof. N.C. Paulescu' National Institute of Diabetes, Nutrition and Metabolic Diseases, 011233 Bucharest; \\ ${ }^{6}$ Dermatology Research Laboratory, 'Carol Davila' University of Medicine and Pharmacy, 050474 Bucharest; \\ ${ }^{7}$ Department of Dermatology, Dermatology Clinic, 'George Emil Palade’ University of Medicine, \\ Pharmacy, Science and Technology, 540139 Târgu Mureș, Romania
}

Received October 19, 2020; Accepted November 18, 2020

DOI: $10.3892 /$ etm.2021.9964

\begin{abstract}
Skin endometriosis is a rare disease with variable clinical and histopathological characteristics that depend on hormonal stimuli. The skin is not a common location, as most cases of endometriosis involve pelvic sites, such as the ovaries, peritoneum and bowel. However, the most common extrapelvic site affected is the abdominal wall and this location of the disease is frequently associated with obstetric and gynecologic surgery. Here we report a case of skin endometriosis emerged as a painful subcutaneous nodule located near to the left side of an obstetrical surgery procedure scar. The patient affected was a woman in her reproductive age, with a history of right ovary endometriotic cyst laparoscopically removed and histologically confirmed as a primary endometriosis. Dermatologists should be aware of this condition in any woman with a painful lump located in the proximity of a pelvic surgery-induced scar. Its non-specific clinical appearance may confuse the clinician and may delay the diagnosis and management.
\end{abstract}

\section{Introduction}

Endometriosis is a gynecological disease affecting fertile women, with a prevalence of $2-10 \%$ in the general female population (1), which can reach up to $50 \%$ in patients with pain, infertility or abdomino-pelvic surgery (2-4). First described by

Correspondence to: Dr Mihaela Adriana Ilie, Dermatology Research Laboratory, 'Carol Davila' University of Medicine and Pharmacy, 8 Eroii Sanitari Street, 050474 Bucharest, Romania

E-mail: mihaelaadriana2005@yahoo.com

${ }^{*}$ Contributed equally

Key words: endometriosis, skin, immunohistochemistry nodule, diagnosis
Rokitansky in 1860 (2), endometriosis is characterized by the presence of endometrial stroma outside the uterus, with the same reactivity to hormonal stimuli as the normal stroma $(3,5)$. The disease can be primary, where the endometrial tissue is found outside the uterus without any external intervention and secondary or iatrogenic, following obstetric and gynecological procedures (uterine wall opening), of which Caesarean-section (C-section) is the most frequent (4).

In patients with $\mathrm{C}$-sections, the incidence can be as high as $1 \%$ (5). Usually, the diagnosis is made several months or up to several years following the procedure (5). The clinical appearance varies with the depth and localization of the tumor (6). The symptomatology is not always present, and the diagnosis is difficult. However, the most frequent symptom is cyclic pain and a positive history for surgery may be a clue for the correct diagnosis $(5,7)$.

Herein, we present the case of skin endometriosis presenting as a subcutaneous nodule in the proximity of a $\mathrm{C}$-section surgical scar and review existing literature in order to increase the index of suspicion in the case of painful lesions appearing close to surgical scars following gynecological or obstetrical procedures.

\section{Case report}

A 29-year-old female presented to the Dermatology Department of 'Prof. Dr. Nicolae C. Paulescu' National Institute of Diabetes, Nutrition and Metabolic Diseases, Bucharest, Romania for the investigation of a painful nodule located in the inferior abdominal wall. Written informed consent was provided and the patient agreed to undergo diagnostic and therapeutic procedures included in the study protocol that was conducted in accordance with the Declaration of Helsinki with approval of the Ethics Committee of the Colentina Clinical Hospital (approval no. 25/27.11.2017).

The patient medical history was significant for a right ovarian endometrial cyst, laparoscopically removed 4 years 
prior to this presentation, and pathologically confirmed as primary endometriosis. The patient presented menstrual cycle-dependent lower abdominal pain after the first surgery. A second laparoscopy was performed, showing multiple sites of endometriosis. The lesions were thermocoagulated followed by treatment with triptorelin $3.75 \mathrm{mg}, 1$ monthly injection for 5 months. After hormonal treatment withdrawal, the patient became pregnant. During pregnancy, the patient experienced diffuse low abdominal pain, but without any complications and she delivered by term $\mathrm{C}$-section. Two years after giving birth, she presented at our department for a painful nodule (the pain was worsening prior menses), located in the lower abdominal wall. The patient also described an intermittent, rather than cyclic, $\mathrm{C}$-section scar pain which started one year after she gave birth.

The physical examination showed a palpable subcutaneous nodule of approximately $1.5 \mathrm{~cm}$ in diameter, round, well defined and mobile, tender on palpation, located $2 \mathrm{~cm}$ superior to the left side of the $\mathrm{C}$-section scar (Pfannenstiel incision). The suprajacent skin was normal. The C-section scar was well-healed, supple and whitish, without any pathological finding upon palpation. A diagnosis of cutaneous endometriosis was suspected given the patient's history of laparoscopic intervention to the ovary and flares of pain with menstrual periods.

The lesion was surgically excised. The macroscopic aspect of the nodule excluded the diagnosis of a cystic lesion or lipoma and the specimen was referred to the pathology department for microscopic examination.

The specimen was routinely processed for paraffinembedding; then 3- $\mu$ m-thick sections were cut and routinely stained with hematoxylin and eosin (H\&E), and immunohistochemistry analysis for CD10, estrogen receptor and Ki67 was performed. The pathologic report showed adipose connective tissue, neuro-vascular and fascial tissue including multiple glandular structures of variable dimensions with a simple columnar focal ciliated epithelium, surrounded by an endometrial stroma (Fig. 1) with multiple hematic extravasations and rare siderophages. The immunohistochemical testing showed positivity for the estrogen receptor in the nuclei of epithelial cells lining glandular structure endometrial-like cells, CD10 diffuse and intense positivity in the endometrial stroma and Ki67 positivity below $1 \%$ of epithelial cell nuclei (Fig. 2). These findings sustained the diagnosis of skin endometriosis. The post-operatory evolution was good, with disappearance of the nodule-associated symptoms. After 1 year, the patient remains without recurrence of the disease or appearance of endometriosis.

\section{Discussion}

Skin endometriosis is a rare disorder with variable clinical and histopathological appearance that depends on hormonal stimuli and it primarily affects women of reproductive age. More than 300 cases of skin endometriosis have been described in the literature and in a review published by Stojanovic et al, they found 210 cases of skin endometriosis located on surgical scars, whereof 119 cases followed a C-section procedure (8).

In regards to skin endometriosis, the most frequent extrapelvic site affected is the abdominal wall and it is associated with obstetric and gynecologic surgery (9). Abdominal wall endometriosis usually presents at surgery departments, being misdiagnosed as incisional hernia or granuloma (10). The clinical differential diagnoses of skin endometriosis are represented by incisional hernia, lipoma, dermoid cyst, abscess, suture granuloma, keloid, melanoma, hematoma and others $(11,12)$.

In our case, based on characteristic history and examination findings, behind the most probable diagnosis of endometriosis, other diagnoses including lipoma, granuloma and desmoid tumor, were also deliberated.

Multiple diagnostic tools have been used in the diagnosis of skin endometriosis. Examination techniques usually used for diagnosis include ultrasonography, computed tomography (CT), magnetic resonance imaging (MRI), and Doppler sonography. Ultrasonography seems to be the first choice for evaluation of any abdominal lesion, but CT and MRI may exclude other possible diagnoses such as a lipoma, hernia or a tumor (13). Tumor markers such as CA19-9 and CA125 may or may not be elevated; however histologic examination after excision of the lesion can confirm the diagnosis (14). Kinkel et al reported the sensitivity and specificity of MRI in diagnosing endometriomas to be $90-92$ and $91-98 \%$, respectively (15).

In regards to skin imaging techniques, standardized dermoscopic features of skin endometriosis have not yet been established. Multiple factors such as the site of occurrence, histological subtype, depth of the lesion or patient phototype may influence the dermoscopic aspect of skin endometriosis (16). However, this examination technique may provide additional information useful for clinical diagnosis. A study published by de Giorgi et al revealed dominant dermoscopic features in skin endometriosis such as homogeneous reddish pigmentation, containing small red globular structures, which they termed 'red atolls' (7). Moreover, Costa et al described the dermoscopy pattern of cutaneous endometriosis in the follicular phase as erythematous-violaceous polypoid projections with light brown spots and areas of active bleeding; further in the luteal phase the dermoscopic feature of the lesion was as an erythematous-bluish aspect (17).

Skin endometriosis usually affects women in their reproductive age (mean age 30-40 years) and its clinical presentation starts with a pigmented or skin-colored papule or nodule with an average diameter of $2 \mathrm{~cm}$. The symptoms associated with this disease are pain, tenderness or bleeding during menstrual cycle and their persistence ranges from 2 months to 2 years (18). After surgery the average period to the onset of symptoms is between 3 months to 18 years (19).

In our case report, there are many similarities to previously reported cases. Our female patient was 29 years of age, thus she was in her reproductive age; the site of the lesion was close to the $\mathrm{C}$-section scar; the lesion appeared approximately 2 years after surgery as a subcutaneous nodule; and associated symptomatology included abdominal pain that started one year after C-section procedure. In this case, characteristic symptoms of endometriosis such as bleeding or monthly swelling were not present and considering previously described differentials near a scar $(20,21)$, this lesion was not easy to diagnose.

The surgical excision revealed a non-cystic appearance, and the specimen was referred to the anatomopathologist. The characteristic features of ectopic endometrial tissue include 

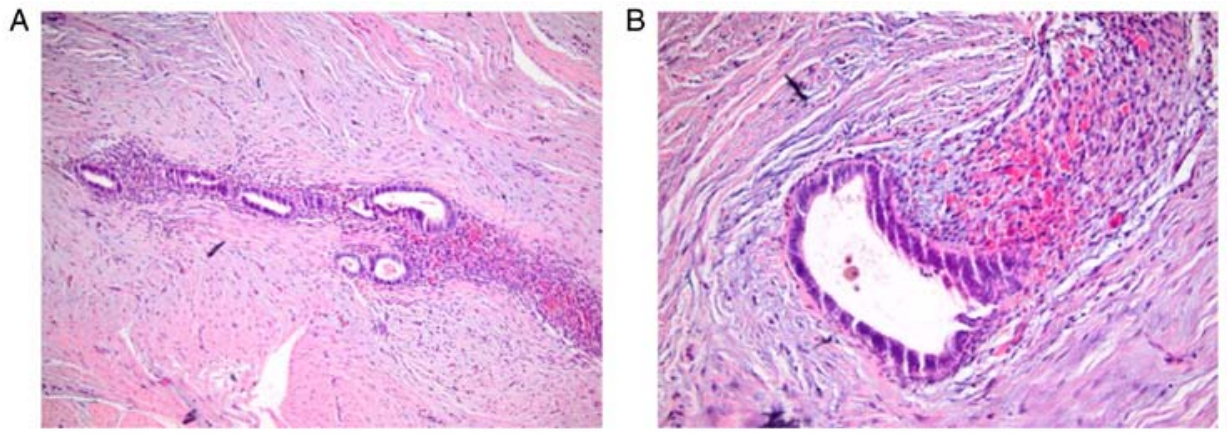

Figure 1. Hematoxylin and eosin-stained histopathological images [(A) (x10 magnification) and (B) (x20 magnification)] showing endometrial glandular structures, bordered by variable amounts of endometrial stroma.
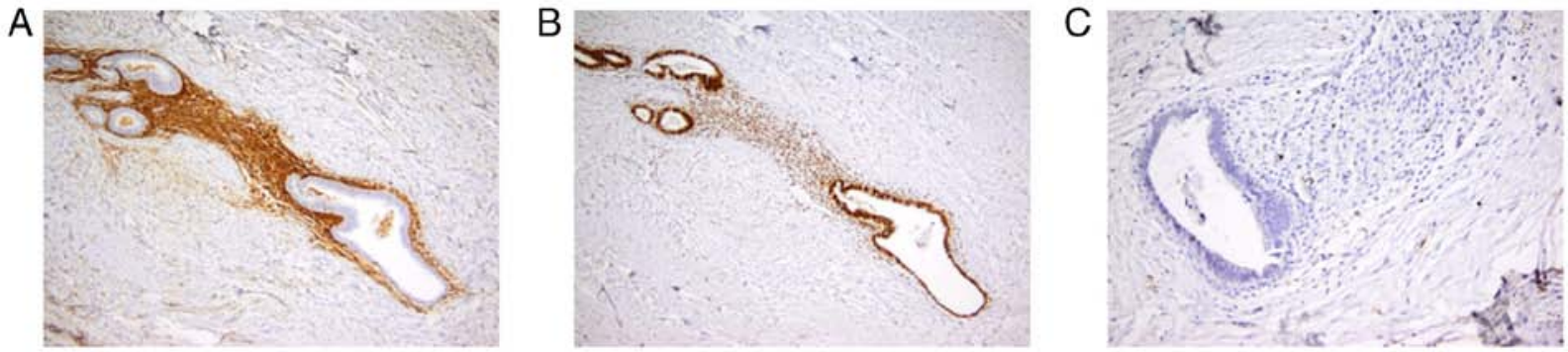

Figure 2. Immunohistochemical staining for (A) CD10 (x10 magnification), (B) estrogen receptor (x10 magnification) and (C) Ki67 (x20 magnification).

glands with cylindrical epithelium and endometrial stroma which may be influenced by cyclic hormonal changes. This is one of the reasons why all stages of the menstrual cycle could be found in the ectopic tissue. Immunohistochemical testing in our case showed positive CD10, a similarity found with other reported cases $(13,22)$. Studies have reported that CD10 as a useful marker for diagnosis as it is strongly expressed in endometrial stromal nodules (23).

Furthermore, in our case, Ki67 positivity was found in less than $1 \%$ of the nuclei of the cells. According to the literature, an increased Ki67 and CD10 positivity indicates that the stroma is decidualized (24). Another finding of our case was the positivity for the estrogen receptor in endometroid-like cells. This was also previously reported (25-28). The production of estrogen can be stimulated in endometrial lesions by aromatase activity (29).

Concerning the possible pathophysiological mechanisms involved, one accepted theory suggests that primitive pluripotent mesenchymal cells that have undergone differentiation metaplasia are one of the cause of abdominal wall endometriosis (30). In addition, some authors propose that retrograde menstruation could be a cause for the implantation of endometrial cells in the peritoneal area (31), while others advocate the theory of lymphatic or hematogenic dissemination (32), the role of genetics (33) and other probable pathophysiological mechanisms $(34,35)$. Another theory explains the possibility that during a $\mathrm{C}$-section procedure endometrial tissue could be iatrogenically grafted into ectopic sites such as the skin, muscles or other layers of the abdominal wall, this being the reason why the nodule appears frequently above the scar. In addition, these grafted endometrial cells are able to proliferate due to hormonal stimulation (36). Therefore, one recommendation according to various authors is to irrigate the abdominal wall with saline solution before closing the abdominal layers (33). Other recommendations published in the literature include isolation of the surgical scar, changing needles during the closure of the superficial layer of the abdomen wall, and replacing the instruments used during C-section to prevent the iatrogenic grafting of cells (37).

A retrospective study of 198 Caesarean scar-related endometriosis cases by Zhang et al found more than $70 \%$ of the endometrial cases in superficial regions of the abdominal wall; $5.7 \%$ were found in the adipose layer and $64.6 \%$ between the adipose layer and the fascia layer, and $83 \%$ were located in a corner of the Pfannenstiel incision scar (38). Ding and Zhu conducted another retrospective study with similar results; $77.1 \%$ of the cases were located in the corners of the scars (39). One argument for this particular location may be that endometrial cells are less easily detached from the corners of the incisions during C-section (38).

The most frequently used abdominal skin incisions are Pfannenstiel incision and vertical midline; therefore $\mathrm{C}$-section seems to be one of the most popular surgical procedures utilized on the female population $(38,39)$. According to Zhang et al, more blood loss in the Pfannenstiel incision would supply a relatively rich nutritional background for the implantation and expansion of residual endometrial cells, facilitating the development of skin endometriosis. Therefore, this type of incision would increase the risk for skin endometriosis (38).

In our patient, the cause for this ectopic tissue may have been the iatrogenic transportation of endometrial cells during the $\mathrm{C}$-section incision. The site of the lesion was found in the superficial abdominal wall, in the proximity of the left side of the scar, after Pfannenstiel incision, as similarly mentioned in the studies above. 
Our treatment for this patient was surgical excision of the lesion with clear margins to prevent recurrence. Generally, surgical excision is the first-line treatment as it is the best way to establish a clear diagnosis and to ensure a low rate of recurrence. The recurrence rate is generally low, but other publications have described recurrence in $6-11 \%$ of patients $(40,41)$. The malignancy rate is also extremely low with less than $1 \%$ of endometriosis cases reported in association with cancer; one of the most common types is clear-cell carcinoma with a survival rate of $80 \%(39,42)$.

Hormonal therapy with gonadotropin releasing hormone (GnRH) agonists, danazol, preoperative or postoperative progesterone is also advocated in the literature. Preoperative therapy is effective in ameliorating symptoms such as pain and minimizing the lesion size. The goal of postoperative hormonal therapy is to prevent recurrence, but its use is still under debate as the overall result has been poor (43). Moreover, considering the psychological and social impact of the disease, patient counseling should also be considered $(44,45)$.

In conclusion, skin endometriosis is a rare and benign condition, with an unknown mechanism and a very low rate of malignancy. Its clinical appearance is very unspecific which may hinder the dermatologist's diagnosis, delaying the right management of the lesion. The rate of C-section is increasing in the female population and the associated incidence in skin endometriosis (found on or in close proximity to a scar associated with this procedure) may increase in the future. Dermatologists should be aware of this condition in any women with pain and a lump close to an incisional scar after pelvic surgery.

The first-line treatment of skin endometriosis is surgical excision and the gold standard for its diagnosis is histopathologic and, if necessary, immunohistochemical examination.

\section{Acknowledgements}

Not applicable.

\section{Funding}

This research and review was funded by a grant from the Romanian Ministry of Research and Innovation, CCCDI-UEFISCDI (project no. 61PCCDI/2018 PN-III-P1-1.2PCCDI-2017-0341) within PNCDI-III.

\section{Availability of data and materials}

All findings generated or analyzed during this study are included in this published article and the literature findings are documented by relevant references.

\section{Authors' contributions}

AMM and AMDI contributed equally to the conceptualization, data analysis, and writing of the manuscript. MC, SAZ, DB, IS and LFG conducted the literature research and data analysis. CC and MAI conducted the data analysis, critical review, editing of the manuscript and supervision of the project. All authors read and gave approval for publication of the final manuscript.

\section{Ethics approval and consent to participate}

Ethics approval was obtained from the Ethics Committee of the Colentina Clinical Hospital (approval no. 25/27.11.2017). Written informed consent of the patient was obtained.

\section{Patient consent for publication}

Written informed consent of the patient was obtained for publication of the information.

\section{Competing interests}

The authors declare that they have no competing interests.

\section{References}

1. Dunselman GAJ, Vermeulen N, Becker C, Calhaz-Jorge C, D'Hooghe T, De Bie B, Heikinheimo O, Horne AW, Kiesel L, Nap A, et al: ESHRE guideline: Management of women with endometriosis. Hum Reprod 29: 400-412, 2014.

2. Giudice LC and Kao LC: Endometriosis. Lancet 364: 1789-1799, 2004.

3. Blanco RG, Parithivel VS, Shah AK, Gumbs MA, Schein M and Gerst PH: Abdominal wall endometriomas. Am J Surg 185: 596-598, 2003.

4. Scholefield HJ, Sajjad Y and Morgan PR: Cutaneous endometriosis and its association with caesarean section and gynaecological procedures. J Obstet Gynaecol 22: 553-554, 2002.

5. Ozel L, Sagiroglu J, Unal A, Unal E, Gunes P, Baskent E, Aka N, Titiz MI and Tufekci EC: Abdominal wall endometriosis in the cesarean section surgical scar: A potential diagnostic pitfall J Obstet Gynaecol Res 38: 526-530, 2012.

6. Tognetti L, Cinotti E, Tonini G, Habougit C, Cambazard F, Rubegni $\mathrm{P}$ and Perrot JL: New findings in non-invasive imaging of cutaneous endometriosis: Dermoscopy, high-frequency ultrasound and reflectance confocal microscopy. Ski Res Technol 24: 309-312, 2018.

7. De Giorgi V, Massi D, Mannone F, Stante M and Carli P: Cutaneous endometriosis: Non-invasive analysis by epiluminescence microscopy. Clin Exp Dermatol 28: 315-317, 2003.

8. Stojanovic M, Brasanac D and Stojicic M: Cutaneous inguinal scar endosalpingiosis and endometriosis: Case report with review of literature. Am J Dermatopathol 35: 254-260, 2013.

9. Bektas H, Bilsel Y, Sar YS, Ersöz F, Koc O, Deniz M, Boran B and Huq GE: Abdominal wall endometrioma; a 10-year experience and brief review of the literature. J Surg Res 164: e77-e81, 2010.

10. Loh SH, Lew BL and Sim WY: Primary cutaneous endometriosis of umbilicus. Ann Dermatol 29: 621-625, 2017.

11. Curry TW: Subcutaneous endometriomas: Two case reports and review of the literature. J Gynecol Surg 14: 31-34, 1998.

12. Tatu AL: Umbilicated blue-black lesion on the lateral thorax. J Cutan Med Surg 21: 252, 2017.

13. Neri I, Tabanelli M, Dika E, Valeria G and Patrizi A: Diagnosis and treatment of post-Caesarean scar endometriosis. Acta Derm Venereol 87: 428-429, 2007.

14. Mechsner S, Bartley J, Infanger M, Loddenkemper C, Herbel J and Ebert AD: Clinical management and immunohistochemical analysis of umbilical endometriosis. Arch Gynecol Obstet 280: 235-242, 2009.

15. Kinkel K, Frei KA, Balleyguier C and Chapron C: Diagnosis of endometriosis with imaging: A review. Eur Radiol 16: 285-298, 2006.

16. Jaime TJ, Jaime TJ, Ormiga P, Leal F, Nogueira OM and Rodrigues N: Endometriose umbilical: Relato de um caso e seus achados dermatoscópicos. An Bras Dermatol 88: 121-124, 2013.

17. Costa IMC, Gomes CM, Morais OO, Costa MC, Abraham LS and Argenziano G: Cutaneous endometriosis: Dermoscopic findings related to phases of the female hormonal cycle. Int $\mathbf{J}$ Dermatol 53: e130-e132, 2014.

18. Friedman PM and Rico MJ: Cutaneous endometriosis. Dermatol Online J 6: 8, 2000. 
19. Kazakov DV, Ondic O, Zamecnik M, Shelekhova KV, Mukensnabl P, Hes O, Dvorak V and Michal M: Morphological variations of scar-related and spontaneous endometriosis of the skin and superficial soft tissue: A study of 71 cases with emphasis on atypical features and types of müllerian differentiations. J Am Acad Dermatol 57: 134-146, 2007.

20. Tatu AL, Kluger N and Nwabudike LC: Pain and shingles on an old scar. Int J Dermatol 59: 1158-1159, 2020

21. Ardeleanu V, Jecan CR, Tatu AL and Motoc AG: A recurrent solitary glomus tumor of the forearm. Rom J Morphol Embryol 60 1019-1023, 2019.

22. Kerr OA, Mowbray M and Tidman MJ: An umbilical nodule due to endometriosis. Acta Derm Venereol 86: 277-278, 2006.

23. Van den Nouland D and Kaur M: Primary umbilical endometriosis: A case report. Facts Views Vis Obgyn 9: 115-119, 2017.

24. Val-Bernal JF, Val D, Gómez-Aguado F, Corcuera MT an Garijo MF: Hypodermal decidualized endometrioma with aberrant cytokeratin expression. A lesion mimicking malignancy. Am J Dermatopathol 33: e58-e62, 2011.

25. Kholová I, Ryska A and Dedic K: Composite tumor consisting of dermatofibrosarcoma protuberans and giant cell fibroblastoma associated with intratumoral endometriosis. Report of a case. Pathol Res Pract 197: 263-267, 269-270, 2001.

26. Dragoumis K, Mikos T, Zafrakas M, Assimakopoulos E, Stamatopoulos P and Bontis J: Endometriotic uterocutaneous fistula after cesarean section. A case report. Gynecol Obstet Invest 57: 90-92, 2004.

27. Frischknecht F, Raio L, Fleischmann A, Dreher E, Lüscher KP and Mueller MD: Umbilical endometriosis. Surg Endosc 18: 347, 2004.

28. Farooq U, Laureano AC, Miteva M and Elgart GW: Cutaneous endometriosis: Diagnostic immunohistochemistry and clinicopathologic correlation. J Cutan Pathol 38: 525-528, 2011.

29. Bulun SE, Imir G, Utsunomiya H, Thung S, Gurates B, Tamura M and Lin Z: Aromatase in endometriosis and uterine leiomyomata. J Steroid Biochem Mol Biol 95: 57-62, 2005.

30. Dwivedi AJ, Agrawal SN and Silva YJ: Abdominal wall endometriomas. Dig Dis Sci 47: 456-461, 2002.

31. Vinatier D, Orazi G, Cosson M and Dufour P: Theories of endometriosis. Eur J Obstet Gynecol Reprod Biol 96: 21-34, 2001.

32. Horton JD, DeZee KJ, Ahnfeldt EP and Wagner M: Abdominal wall endometriosis: A surgeon's perspective and review of 445 cases. Am J Surg 196: 207-212, 2008.
33. Witz CA. Pathogenesis of endometriosis. Gynecol Obstet Invest 53 (Suppl 1): S52-S62, 2002.

34. Nwabudike LC and Tatu AL: Reply to Happle R et al. Koebner's sheep in Wolf's clothing: Does the isotopic response exists as a distinct phenomenon? J Eur Acad Dermatology Venereol 32: e336-e337, 2018.

35. Aida Maranduca M, Liliana Hurjui L, Constantin Branisteanu D, Nicolae Serban D, Elena Branisteanu D, Dima N and Lacramioara Serban I: Skin-a vast organ with immunological function (Review). Exp Ther Med 20: 18-23, 2020.

36. Seydel AS, Sickel JZ, Warner ED and Sax HC: Extrapelvic endometriosis: Diagnosis and treatment. Am J Surg 171: 239-241, 1996.

37. Agarwal A and Fong YF. Cutaneous endometriosis. Singapore Med J 49: 704-709, 2008.

38. Zhang P, Sun Y, Zhang C, Yang Y, Zhang L, Wang N and Xu H: Cesarean scar endometriosis: Presentation of 198 cases and literature review. BMC Womens Health 19: 14, 2019.

39. Ding Y and Zhu J: A retrospective review of abdominal wall endometriosis in Shanghai, China. Int J Gynecol Obstet 121: 41-44, 2013.

40. Chatterjee SK: Scar endometriosis: A clinicopathologic study of 17 cases. Obstet Gynecol 56: 81-84, 1980

41. Steck WD and Helwig EB: Cutaneous endometriosis. JAMA 191: $167-170,1965$.

42. Petca A, Radu N and Petca R: Insights into malignant potential of ovarian endometriomas. In: The 17th National Congress of the Romanian Society of Obstetrics and Gynecology and First Advanced Colposcopy Course. pp614-619, 2019.

43. Raffi L, Suresh R, McCalmont TH and Twigg AR: Cutaneous endometriosis. Int J Womens Dermatol 5: 384-386, 2019.

44. Rebegea L, Firescu D, Baciu G and Ciubara A: Psycho-oncology support. BRAIN Broad Res Artif Intell Neurosci 10: 77-88, 2019.

45. Aerts L, Grangier L, Streuli I, Dällenbach P, Marci R, Wenger JM and Pluchino N: Psychosocial impact of endometriosis: From co-morbidity to intervention. Best Pract Res Clin Obstet Gynaecol 50: 2-10, 2018. 